\title{
INDEX SETS AND BOOLEAN OPERATIONS ${ }^{1}$
}

\author{
DOUGLAS E. MILLER
}

\begin{abstract}
Hay's thesis asserts that every naturally defined class of sets of natural numbers contains an index set which is 1-complete for that class and that every index set is 1-complete for some naturally defined class. We formalize "naturally defined" as "effective Boolean" and establish the thesis as a Metatheorem.
\end{abstract}

Introduction. An index set is a set of natural numbers closed under the equivalence $m \sim n \Leftrightarrow W_{m}=W_{n}$, where $\left\langle W_{i}: i \in \omega\right\rangle$ is the canonical listing of all recursively enumerable (r.e.) sets. The most familiar index set is the complete r.e. set $K=\{n$ : $\left.W_{n} \neq \varnothing\right\}$. It follows from the Rice and Rice-Shapiro theorems (e.g. [10]) that any other properly r.e. index set is recursively isomorphic to $K$ and hence is also 1-complete for the class $\Sigma_{1}^{0}$.

That situation is typical. Consider for example the relativized arithmetical classes $\Sigma_{m}^{A}, \Pi_{m}^{A}$ and small arithmetical classes $\Sigma_{m, k}^{A}, \Pi_{m, k}^{A}$ (notation as in [3]). Each of these classes contains an index set which is 1-complete for the class. Virtually every arithmetical index set in the literature has been classified as complete for one of these classes. This phenomenon led Hay to informally promote the thesis stated in the abstract and has motivated a good deal of her work. See e.g. [3 and 5]. In fact, the relativized difference classes were introduced into the literature in [3] to solve the problem of classifying certain index sets.

Recently she asked (privately) what "natural classes" corresponded to the index sets $X_{n}^{\beta}$ described in [4]. Our Metatheorem answers that question and all questions of the same sort. (Her specific question is discussed further in Remark II below.) The basic ideas and combinatorial method used in the proof of our Metatheorem may be found in Ershov [1, 2] and Hay [3].

The author is indebted to Robert Soare, Jeanleah Mohrherr and, especially, Louise Hay for comments and lessons on the subject discussed herein.

Effective Boolean classes. The notion of a finitary Boolean set operation and the associated disjunctive normal form for such goes back to Boole and Post. Infinitary set operations were introduced as "analytical operations" by Kantorevich and Livenson [8]. More recently, they have been discussed in seminars at Berkeley (notably led by J. W. Addison), in several dissertations [6, 11, 12], and in Hinman's book [7].

Received by the editors April 27, 1981.

1980 Mathematics Subject Classification. Primary 02F25, 02F35; Secondary 02K30, 04A15.

Key words and phrases. Index sets, many-one degrees, hierarchies, Boolean set operations.

${ }^{1}$ Research partially supported by NSF Grant \#78-02845. 
Effective $\omega$-Boolean classes are the recursion-theoretic analogs to the classes $\boldsymbol{\aleph}_{N}(\mathbb{Q})$ introduced by Kantorevich and Livenson. While examples of effective Boolean classes abound, the general notion (particularly in the case of nonpositive operations) has received little attention. A review of the definitions follows.

Given an indexed family of sets $\left\langle A_{i}: i \in I\right\rangle$ and an element $x$ let: $\chi_{\left\langle A_{i}\right\rangle}^{x}$ be the characteristic function of $x$ with respect to the family $\left\langle A_{i}\right\rangle$, so

$$
\chi_{\left\langle A_{i}\right\rangle}^{x}(j)=\left\{\begin{array}{ll}
1 & \text { if } x \in A_{j}, \\
0 & \text { otherwise. }
\end{array} .\right.
$$

Let $B \subseteq 2^{I}$. The $I$-Boolean set operation based on $B$ is the map $\Gamma$ which sends each family $\left\langle A_{i}: i \in I\right\rangle$ to the set $\Gamma\left\langle A_{i}\right\rangle=\left\{x: \chi_{\left\langle A_{i}\right\rangle}^{x} \in B\right\}$. Thus, for example, complementation, countable intersection, and the operation $(Q)$ can be represented as $\omega$-Boolean operations based on the sets $B_{0}=\left\{\xi \in 2^{\omega}: \xi(0)=0\right\}, B_{1}=\left\{\xi \in 2^{\omega}\right.$ : $(\forall n)(\xi(n)=1)\}, B_{2}=\left\{\xi \in 2^{\omega}: \exists f \in \omega^{\omega} \forall n(\xi\langle f \mid n\rangle=1)\right\}$ where \langle\rangle$: \omega^{\omega} \rightarrow \omega$ is the inverse of a standard enumeration of all finite sequences of natural numbers.

The following two observations about set operations are useful. Let $\Gamma$ be any set operation; then

(1) For every family $\left\langle A_{i}\right\rangle$ and every function $f, f^{-1}\left(\Gamma\left\langle A_{i}\right\rangle\right)=\Gamma\left\langle f^{-1} A_{i}\right\rangle$.

(2) If $\mathrm{E}$ is any equivalence relation and each $A_{i}$ is E-closed $\left(\left(x \in A_{i} \& y \mathrm{E} x\right) \Rightarrow y \in\right.$ $\left.A_{i}\right)$, then $\Gamma\left\langle A_{i}\right\rangle$ is E-closed.

Now consider the standard indexing (as found, e.g., in [10]) of the collection of all recursively enumerable sets as $\left\{W_{n}: n \in \omega\right\}$. Given a function $f: \omega \rightarrow \omega$ and an $\omega$-Boolean operation $\Gamma$, write $\Gamma(f)=\Gamma\left\langle W_{f(n)}: n \in \omega\right\rangle . \Phi_{\Gamma}$, the effective Boolean class determined by $\Gamma$, is defined by

$$
g_{\Gamma}=\{\Gamma(f): f \text { is recursive }\} .
$$

Thus, for example, the operations complementation, countable intersection, and operation $(Q)$ respectively determine the effective classes $\Pi_{1}^{0}, \Pi_{2}^{0}, \Sigma_{1}^{1}$. It is not difficult to show that each of the classes mentioned in the introduction is an effective Boolean class. (For the unrelativized classes this is obvious; for the relativized case see Remark I below.)

In the special case of a positive Boolean operation $\Gamma$ with dual $\Gamma^{0}, q_{\Gamma}$ corresponds to Hinman's $\Pi_{1}^{\Gamma^{0}}$ (see [7] for definitions).

A collection $\mathscr{\Phi} \subseteq \mathcal{P}(\omega)$ is $m$-closed provided $(\forall A, B)\left(\left[A \in \mathscr{Q} \& B \leqslant_{m} A\right] \Rightarrow B \in \mathscr{q}\right)$. $A$ is $m$-complete (resp. 1-complete) for $\mathscr{G}$ provided $A \in \mathscr{G} \&(\forall B \in \mathscr{G})\left(B \leqslant_{m} A\right)$ (resp., $\left.(\forall B \in \mathscr{G})\left(B \leqslant_{1} A\right)\right)$. We write $\mathscr{G}_{A}=\left\{B: B \leqslant_{m} A\right\}$. Clearly $\mathscr{q}_{A}$ is $m$-closed. Note that $A$ is $m$-complete for $\mathscr{G}$ iff $\mathscr{G}=\mathscr{G}_{A}$. It is known [2, p. 213] that if $A$ is an index set, then $A$ is 1-complete for $\mathscr{G}_{A}$ and the 1-degree of $A$ is an $m$-degree. It follows that $A$ is characterized up to recursive isomorphism as the $m$-complete set for $\oint_{A}$.

It follows from (1) that

(3) For any set operation $\Gamma$, the associated effective class $\Phi_{\Gamma}$ is $m$-closed.

It follows from (2) that the application of any set operation to a family of index sets yields another index set. 
Following Hay [3] we write, for $k \in \omega$,

$$
\mathfrak{T}_{k}=\left\{n: k \in W_{n}\right\} \text {. }
$$

We may fix a recursive function $f_{0}$ such that for all $k, \mathfrak{R}_{k}=W_{f_{0}(k)}$. Note that $\left\langle\mathscr{N}_{k}\right\rangle$ is a family of index sets and that $\Gamma\left\langle\mathscr{N}_{k}\right\rangle=\Gamma\left(f_{0}\right) \in \mathscr{G}_{\Gamma}$.

\section{The Metatheorem.}

THEOREM. (a) Given any $\omega$-Boolean set operation $\Gamma$, the index set $\Gamma\left\langle\Re_{k}\right\rangle$ is 1-complete for the associated effective class $\Phi_{\Gamma}$.

(b) Given any index set $A$, there is an $\omega$-Boolean set operation $\Gamma$ such that $A$ is 1-complete for $\Phi_{\Gamma}$.

Proof of (a) (cf. Ershov [2, Theorem 2] and Hay [3, Theorem 7.2]). Fix $\Gamma$ and suppose $A \in \mathscr{I}_{\Gamma}$; say $A=\Gamma\left\langle W_{g(n)}\right\rangle$ with $g$ recursive. Fix a recursive $h$ such that $\forall m\left(W_{h(m)}=g^{-1} \Re_{m}\right)$. Then for any $m$,

$$
m \in W_{g(n)} \Leftrightarrow g(n) \in \Re_{m} \Leftrightarrow n \in W_{h(m)} \Leftrightarrow h(m) \in \mathfrak{T}_{n} .
$$

Thus, $\left.\chi_{\left\langle W_{g(n)}\right\rangle}^{m}=\chi_{\langle\mathscr{N}}^{h(m)}\right\rangle$. It follows that $m \in A \Leftrightarrow h(m) \in \Gamma\left\langle\mathscr{R}_{n}\right\rangle$, so $h$ is an $m$ reduction of $A$ to $\Gamma\left\langle\mathscr{T}_{n}\right\rangle$. Thus, $\Phi_{\Gamma}=\Phi_{\Gamma\left\langle\mathscr{N}_{n}\right\rangle}$. As previously remarked, $\Gamma\left\langle\mathscr{T}_{n}\right\rangle$ is 1-complete for $\Phi_{\Gamma\left\langle\pi_{n}\right\rangle}$.

Proof of (b) (cf. Ershov [1, Assertion 2]). For any $R \subseteq \omega$, let $\hat{R} \in 2^{\omega}$ be the charcteristic function of $R$ (so $\hat{R}(n)=1$ iff $n \in R$ ). Given $C \subseteq \mathcal{P}(\omega)$, let $\hat{C}=$ $\{\hat{R}: R \in C\}$ and let $\Gamma_{C}$ be the $\omega$-Boolean operation based on $\hat{C}$. Note that for any sequence $\left\langle A_{i}: i \in \omega\right\rangle$,

$$
\Gamma_{C}\left\langle A_{i}\right\rangle=\bigcup_{R \in C}\left(\bigcap_{k \in R} A_{k} \cap \bigcap_{k \notin R}\left(\overline{A_{k}}\right)\right) .
$$

Now fix an index set $A$ and let $C=\left\{W_{n}: n \in A\right\}$. By (a) it suffices to show that $A=\Gamma_{C}\left\langle\mathfrak{T}_{k}\right\rangle$. We compute

$$
\begin{aligned}
n \in A & \Leftrightarrow(\exists R \in C)\left(W_{n}=R\right) \\
& \Leftrightarrow(\exists R \in C)(\forall k)\left(k \in W_{n} \Leftrightarrow k \in R\right) \\
& \Leftrightarrow(\exists R \in C)(\forall k)\left(\left[k \in R \Rightarrow n \in \mathbb{N}_{k}\right] \&\left[k \notin R \Rightarrow n \notin \mathscr{N}_{k}\right]\right) \\
& \Leftrightarrow(\exists R \in C)\left(n \in \bigcap_{k \in R} \Re_{k} \cap \bigcap_{k \notin R}\left(\overline{\mathscr{N}}_{k}\right)\right) \\
& \Leftrightarrow n \in \Gamma_{C}\left\langle\mathfrak{R}_{k}\right\rangle . \quad \square
\end{aligned}
$$

COROLlary. Every effective Boolean class contains a set which is 1-complete for the class and whose 1-degree is an m-degree.

Remarks. I. Given any $A$ let $\cup_{A}$ be the operation defined by $\cup_{A}\left\langle B_{i}\right\rangle=\cup_{i \in A} B_{i}$. The complete set for $\Phi_{U_{A}}$ is

$$
H_{A}=\bigcup_{A}\left\langle\mathscr{N}_{i}\right\rangle=\left\{i: W_{i} \cap A \neq \varnothing\right\} .
$$


$H_{A}$ is familiar as the weak jump of $A$. Writing $A^{\prime}$ for the ordinary jump of $A$, and $\leqslant_{e}$ for "enumeration reducible to", it can be seen that $H_{A} \leqslant_{e} A$ and hence $H_{A^{\prime}} \equiv_{1} A^{\prime}$. This provides a simple way to obtain complete index sets for the relativized arithmetical classes $\Sigma_{n}^{A}$, etc. Given an operation generating a class $\Sigma_{n}^{A}$, it is then straightforward to construct operations generating the finite or transfinite difference classes $\Sigma_{n, m}^{A}, \Sigma_{n, a}^{A}$. ( $\Sigma_{n, a}^{A}$ is to be read as a generalization of Ershov's $\Sigma_{a}^{-1}\left(=\Sigma_{1, a}^{0}\right)$, where $a$ is an index for a recursive ordinal.)

II. Let $A$ be any index set and suppose $\Gamma$ is chosen such that $A=\Gamma\left\langle\mathscr{N}_{i}\right\rangle$. Then by the Metatheorem, $\mathscr{G}_{\Gamma}$ is the effective Boolean class associated with $A$. Using this remark, we can sometimes provide a simpler description of $\Gamma$ than that provided by part (b) of the Metatheorem. Consider, for example, the index sets introduced in Hay [4]. Given an arbitrary enumerated set of natural numbers $\beta=\left\{b_{i}: i \in \omega\right\}$, the sets $X_{n}^{\beta}$ are defined recursively by

$$
\begin{gathered}
X_{0}^{\beta}=\left\{x: W_{x} \subseteq B\right\}=\bigcap_{k \in \bar{\beta}} \overline{\mathfrak{M}}_{k}, \\
X_{2 n+1}^{\beta}=X_{2 n}^{\beta} \cap \Re_{b_{2 n}}, \quad X_{2 n+2}^{\beta}=X_{2 n+1}^{\beta} \cup \bar{\Re}_{b_{2 n+1}} .
\end{gathered}
$$

Thus, $X_{0}^{\beta}$ is the complete set for the effective class determined by the operation $\left\langle A_{i}\right\rangle \mapsto \bigcap_{k \in \bar{\beta}} \overline{A_{i}} . X_{1}^{\beta}$ is complete for the class associated with $\left\langle A_{i}\right\rangle \mapsto \bigcap_{k \in \bar{\beta}} \overline{A_{i}} \cap$ $A_{b_{0}} \cdot X_{2}^{\beta}$ is complete for $\left\langle A_{i}\right\rangle \mapsto\left(\cap_{k \in \bar{\beta}} \bar{A}_{i} \cap A_{b_{0}}\right) \cup \overline{A_{b_{1}}}$; etc. The classes would appear somewhat more natural if the sets $\bar{X}_{n}^{\beta}$ were taken as primitive. For example $\bar{X}_{0}^{\beta}$ is just $H_{\bar{\beta}}$ and the associated operation is just $\cup_{\bar{\beta}}$.

III. It is interesting to note the connection between $X_{0}^{\beta}$ and $Q$-reducibility (cf. Odifreddi [9, p. 48]). Succinctly, $A \leqslant_{Q} B$ iff $A \leqslant_{m} X_{0}^{\beta}$. Thus, $X_{0}^{\beta}$ is the 1-complete set for the class $\left\{A: A \leqslant{ }_{Q} B\right\}$.

Dualizing, we could define a reducibility $\leqslant_{U}$ by writing $A \leqslant_{U} B \Leftrightarrow W_{f(u)} \cap B \neq$ $\varnothing$. Succinctly, $A \leqslant_{U} B \Leftrightarrow A \leqslant_{m} H_{B}$. (Note that $\leqslant_{U}$ is transitive so this is a genuine reducibility.) Clearly, one can generate families of reduction relations by replacing the operation $\cup_{A}$ by other Boolean operations replacing $A$ by another set depending on $A$. Note, for example, the equivalences

$$
A \leqslant_{e} B \Leftrightarrow A \leqslant_{U} B^{*}
$$

and

$$
A \text { r.e. in } B \Leftrightarrow A \leqslant{ }_{U} B^{\circledast},
$$

where $B^{*}=\{\langle s\rangle:$ range $(s) \subseteq B\}$, and $B^{\circledast}=\{\langle s\rangle: s$ is an initial segment of $\hat{B}\} . \leqslant_{T}$ denotes Turing reducibility.

\section{REFERENCES}

1. Y. L. Ershov, Completely enumerated sets, Algebra and Logic 9 (1970), 20-31.

2. __ On a hierarchy of sets. II, Algebra and Logic 7 (1968), 212-232.

3. L. Hay, Index sets universal for differences of arithmetic sets, Z. Math. Logik Grundlag. Math. 20 (1974), 239-254.

4. _ A noninitial segment of index sets, J. Symbolic Logic 39 (1974), 209-224.

5. L. Hay, A. Manaster and J. Rosenstein, Small recursive ordinals, many one degrees, and the arithmetical difference hierarchy, Ann. Math. Logic 8 (1975), 297-343. 
6. P. Hinman, Ph. D. Dissertation, Univ. of California, Berkeley, 1966.

7. __ Recursion theoretic hierarchies, Springer-Verlag, Berlin, 1978.

8. L. Kantorevich and E. Livenson, Memoir on the analytical operations and projective sets, Fund. Math. 18 (1932), 214-279 and 20 (1933), 54-97.

9. P. Odifreddi, Strong reducibilities, Bull. Amer. Math. Soc. 4 (1981), 37-86.

10. H. Rogers, Jr., Theory of recursive functions and effective computability, McGraw-Hill, New York, 1967.

11. J. Steel, Ph.D. Dissertation, Univ. of California, Berkeley, 1977.

12. R. Van Wesep, Ph.D. Dissertation, Univ. of California, Berkeley, 1977.

Department of Mathematics, University of Illinois at Chicago Circle, Chicago, Illinois 60680

Current address: Schlumberger-Doll Research, Old Quarry Road, Ridgefield, Connecticut 06877 